\title{
UTOPIES ET COSMOPOLITISME
}

\author{
EMF : Studies in Early Modern France. Vol. IV : Utopia 1. Sixteenth and seventeenth \\ centuries; vol. V : Utopia 2. The eighteenth century. Ed. by David LEE RUBIN, \\ co-editor Alice Stroup. Charlottesville, VA, Rookwood Press, 1998 et 1999. \\ $15,5 \times 23,5$, XII- 224 p., et X-131 p., index (EMF : Studies in Early Modern \\ France).
}

La série de recueils d'études sur la France de 1500 à 1800 publiée aux ÉtatsUnis, Studies in Early Modern France, consacre ses quatrième et cinquième volumes à l'utopie. Le travail préparatoire de définition du cadre général de ces études de l'utopie française des $\mathrm{XVI}^{\mathrm{e}}, \mathrm{XVII}^{\mathrm{e}}$ et XVIII ${ }^{\mathrm{e}}$ siècles est assuré par Alice Stroup, dans son article «French utopian thought. The culture of criticism ». Elle rappelle qu'en matière d'utopie, «si l'Angleterre peut se targuer de l'invention, et Athènes de l'inspiration, de tous les pays, c'est la France qui publia le plus après 1650 » (vol. IV, p. 1). Elle note également que «se concentrer sur la pensée utopique française, c'est paradoxalement reconnaître son caractère à la fois national et international. La langue et l'objet satirique caractérisent les œuvres utopiques comme françaises, dans la mesure où celles-ci illustrent une expérience et une culture partagées, qu'elles soient imprimées en France par Cyrano et Fontenelle ou à l'étranger par des expatriés huguenots tels Foigny ou Simon Tyssot de Patot» (vol. IV, p. 9).

La chronologie et l'aire étant fixées, reste le problème, propre à toute recherche sur l'utopie, de sa définition conceptuelle. Quelle position adopter en effet vis-à-vis de la distinction traditionnellement faite entre le genre utopique d'une part (et l'histoire littéraire qui en suit les avatars), et le mode utopique d'autre part (dont l'examen philosophique, sociologique et politique relève d'une histoire des idées) ? Pour Stroup, « [b]ien que l'utopisme soit mieux connu comme un artefact littéraire incarné dans les innombrables écrits romanesques publiés entre 1516 et la Révolution française, celui-ci fonctionnait plus comme un style de pensée critique et subversive que comme un genre littéraire en tant que tel» (vol. IV, p. 6). « La pensée utopique était plus un tour d'esprit qu'un genre littéraire. C'était une forme de pensée critique et subversive qu'employaient les lecteurs et les écrivains, soit indépendamment les uns des autres, soit de concert, surtout lorsqu'ils étaient inspirés par des pensées de voyage » (vol. IV, p. 18). On pense à l'esthétique de la réception en lisant cet essai liminaire, lorsque Stroup montre, à partir d'une analyse des comptes rendus et de la classification des ouvrages utopiques dans La Bibliothèque françoise de Charles Sorel (1664-1667), le Journal des sçavants (à partir de 1665), et les Voyages imaginaires de Charles-Georges-Thomas Garnier (1787-1789), comment le genre utopique a été constitué conjointement par l'écriture et par la 
lecture. Partant du genre utopique en formation, Stroup ouvre donc sur le mode utopique : «Pendant les seizième et dix-septième siècles, les écrivains commencèrent à élaborer un style littéraire caractéristique qui devint un véhicule de dissenssion » (vol. IV, p. 20). D'où la portée générale de l'interprétation des récits de voyages imaginaires, si importants dans l'utopie du XVII siècle : «[...] réfléchir à des mondes différents, c'est s'interroger sur sa propre société » (vol. IV, p. 20).

Rappelant les caractéristiques de l'utopie française de la Renaissance aux Lumières, et fournissant un cadre délibérément large à son étude - outrepassant en cela les limites assignées à l'utopie narrative stricto sensu par Jean-Michel Racault, dans L'Utopie narrative en France et en Angleterre, 1675-1761 (Oxford, Voltaire Foundation, 1991) -, la première contribution de Stroup peut être lue comme une introduction méthodologique à l'ensemble que forment ces deux volumes : « [...] la pensée utopique n'était pas coextensive à l'écriture de fictions ou à l'interprétation allégorique. Aucun recensement de la littérature sur des voyages et des lieux imaginaires ne peut faire justice à la pensée utopique. Bien plutôt, il faut prendre en compte une gamme d'expression plus large qui engloberait la société des amis selon Étienne de la Boétie, le commentaire pictural de Nicolas Poussin, la communauté religieuse de la visionnaire Antoinette Bourignon, le plan colonial du Huguenot Henri Duquesne, les projets de paix de l'abbé de Saint-Pierre, ou les complots politiques de Philippe de Gentil, le roué marquis de Langalerie. L'utopisme de la France de l'âge classique impliquait la lecture, l'écriture, les discussions et l'action. Il ne se limite pas à la production de textes, mais comprend une demande de critique et de réforme de l'Ancien Régime » (vol. IV, p. 6).

C'est dans cette perspective que s'inscrivent l'article de Twyla A. Meding ( «Diana's domain. The displaced center of feminine utopia in Honoré d'Urfé's L'Astrée ») qui montre que « la description initiale du lieu et la prédominance de certaines des caractéristiques majeures de l'utopie suggèrent que sans être à proprement parler une utopie, L'Astrée participe plus généralement d'un "mode de pensée" utopique » (vol. IV, p. 88), ainsi que deux études sur la dialectique de l'utopique et du dystopique, dans la mise en parallèle d'Étienne de La Boétie et de Thomas Hobbes, dans l'œuvre de René Descartes et dans deux tableaux de Nicolas Poussin. Timothy J. Reiss, dans «Utopie versus état de pouvoir, ou prétexte du discours politique de la modernité. Hobbes, lecteur de La Boétie», retrace la généalogie bipolaire du discours politique moderne en étudiant la «communauté d'amitié » dans le Discours de la servitude volontaire en relation avec l'œuvre de Hobbes. Laissant de côté Les Bergers d'Arcadie ( Et in Arcadia ego »), et le versant politique de l'œuvre de Descartes, Van Kelly étudie l'analogie entre l'idéal d'ordre à l'œuvre dans la physique et la métaphysique cartésiennes, c'est-à-dire « la représentation utopique que se fait Descartes du moi et du monde [qui] se caractérise par l'importance donnée à l'harmonie, à la constance, à l'évidence et à l'unanimité » (vol. IV, p. 39), et la notion classique d'eurythmie autour de laquelle Poussin a peint le Paysage avec les funérailles de Phocion et le Paysage avec les cendres de Phocion recueillies par sa veuve en 1647-1648 («The play of utopia and dystopia. Mindscape and landscape in Descartes and Poussin »). 
Alice Stroup exerce ensuite sa méthode d'analyse en explicitant les idées philosophiques à l'œuvre dans La Terre australe connue (1676) de Gabriel Foigny («Foigny's joke»). Carole Martin relit, pour clore ce premier volume, l'Histoire des Sévarambes (1677-1679) de Denis Veiras («L'Utopie, le souverain et l'individu. Le cas des Sévarambes »), assurant par là une transition avec le volume consacré au XVIII ${ }^{\mathrm{e}}$ siècle. La question de la classification des utopies se repose, lorsque Martin revient sur ce qu'elle appelle l' « angle mort », la « solution de continuité », l' « ellipse », l' « impasse sur le siècle de Louis XIV » (vol. IV, p. 195) qu'elle relève dans les études et les colloques sur l'utopie lors des cinquante dernières années. Pour Martin, si l'œuvre de Veiras a été lue souvent comme une œuvre du XVIII ${ }^{\mathrm{e}}$ siècle, c'est par un effet d' « absorption d'une image de l'absolutisme en les préparatifs de son abolition » (vol. IV, p. 197).

Outre une relecture des Voyages et avantures de Jacques Massé (1710) de Simon Tyssot de Patot par Stroup («Massé's haircut»), et un réexamen de «l'attitude ambivalente de Rousseau à l'égard de l'utopie» (vol. V, p. 60) par Peter G. Stillman ( «topia and anti-utopia in Rousseau's thought»), le volume V des Studies in Early Modern France retrace les péripéties de la singulière utopie huguenote de la théocratie du Verbe incarné (John O’Connor, « Exploitation and subversion of utopian ideals. The schemes of two french exiles in the Netherlands »). Place est faite, enfin à ceux à propos desquels Raymond Trousson rapporte dans ses Voyages aux pays de nulle part qu'ils « ont porté la fantaisie jusqu'à la divagation » (Éditions de l'université de Bruxelles (1975), ici 1999, p. 170) : Nicolas Restif et Giovanni Giacomo Casanova. Clifford D. Conner s'intéresse aux rapports entre l'utopie et les conceptions scientifiques de certains membres de la confédération des Amis de la vérité, dont en particulier l'auteur de La Découverte australe par un homme volant («Utopia, natural philosophy, and the Cercle social. The sexual universe of Restif de La Bretonne »). Lise Leibacher-Ouvrard (« L'Icosameron de Casanova. Nature et culture de l'ambiguïté ») s'engage dans les voies souterraines des cinq volumes de l'Icosameron (1788), pour creuser la question de la « pratique originale de l'utopie » de son auteur (vol. V, p. 104).

Ces deux volumes des Studies in Early Modern France reconstruisent une séquence capitale de l'histoire de l'utopie, dont ils soulignent les nombreuses articulations. Par leur diversité, les études qui y sont présentées montrent la difficulté inhérente à toute tentative de classement des utopies en même temps que leur relative cohérence, tout à la fois la permanence de certains thèmes et les nombreuses variations qui en découlent. Par là, elles illustrent bien la «complexité du mode utopique » (Leibacher-Ouvrard, vol. V, p. 110), aident à mesurer l'entière tessiture de l'utopie et attestent de cette large « gamme d'expression » qu'elle rend possible.

Antoine HATZENBERGER 
Bettina DIETZ, Utopien als mögliche Welten. Voyages imaginaires der französischen Frühaufklärung (1650-1720). Mayence, Philipp von Zabern, 2002. 17,5 × 24,5, VIII- 250 p., biogr., bibliogr., index (Veröffentlichungen des Instituts für Europäische Geschichte Mainz, Abteilung für Universalgeschichte, Bd. CLXXXVIII).

Bettina Dietz publie une version allégée de sa thèse de doctorat en histoire, soutenue à l'université Louis-Maximilien de Munich en 2000. L'ouvrage s'organise en deux parties, de respectivement deux et trois chapitres : la première est consacrée au concept d'État idéal, la seconde à l'information et à la circulation des idées et des opinions en France autour de 1700. L'auteur se propose d'étudier les récits de voyages utopiques français publiés entre 1650 - alors que débute l'ère des « voyages imaginaires » - et 1720 - les Lettres persanes (1721) marquent selon Dietz un tournant et l'avènement d'un genre nouveau. Sur le plan politique, la période correspond donc pour l'essentiel au règne de Louis XIV. L'approche est classique : l'utopie est fondamentalement une histoire de communication, de circulation harmonieuse dans l'espace géographique, mais aussi social, religieux, urbain, privé et public, et dans le temps, capable de retisser un lien social et politique à la fois solide et souple, que le système louis-quatorzien (Dietz utilise à maintes reprises l'image de la «constellation» louis-quatorzienne) aurait mis à mal. Le récit de voyage utopique est un «art du gouvernement». La réflexion sur les institutions à mettre en place pour assurer l'ordre dans l'harmonie et l'équilibre conduit à développer une véritable science du gouvernement, et ce faisant à juger à l'aune de ses acquis les tensions qui éprouvent et meurtrissent la société française. Le corpus de récits de voyages réuni à l'appui de cette thèse est étoffé, même si Dietz étudie principalement La Terre australe connue de Gabriel de Foigny (Genève, 1676), Les Aventures de Jacques Sadeur dans la découverte et le voyage de la terre australe (Paris, 1692) du même auteur, La République des philosophes ou histoire des Ajaoiens de Fontenelle (Amsterdam, 1768), l'Histoire de Calejava ou de l'île des hommes raisonnables, avec le parallèle de leur morale et du christianisme (Dijon, 1700) de Claude Gilbert, les Nouveaux voyages de M. le baron de Lahontan dans l'Amérique septentrionale de Louis Armand de Lom d'Arce, baron de La Hontan (La Haye, 1703), les Voyages et aventures de Jacques Massé, œuvre de Simon Tyssot de Patot (Dijon, 1710), l'Histoire des Sévarambes, peuples qui habitent une partie du troisième continent communément appelée la terre australe. Contenant un compte exact du gouvernement, des mours, de la religion et du langage de cette nation jusqu'aujourd'hui inconnue aux peuples de l'Europe de Denis Veiras (Paris, 1677). Une courte biographie de chaque auteur est proposée en annexe. Dietz souligne que les récits de voyages utopiques souffrent d'une dévalorisation symbolique - Paul Hazard les taxant notamment de « bric-à-brac »-due à la difficulté de les classer dans un domaine disciplinaire : histoire, géographie, littérature, sciences et techniques, ethnographie, anthropologie. Seuls les textes de Fontenelle et de Fénelon sont largement connus. La bibliographie proposée, riche et à jour, complète utilement le corpus de récits.

Même s'ils appartiennent au genre des voyages imaginaires, ces récits se présentent comme des relations de voyages authentiques. Leurs auteurs s'appuient 
sur les découvertes et les récits d'expédition du moment ainsi que sur la thèse de l'existence d'autres planètes, d'autres « mondes possibles » pour faire écho au titre de l'ouvrage de Dietz qui s'ouvre sur une citation de Christian Huyghens à propos de son Nouveau traité de la pluralité des mondes (1702) et de Fontenelle (Entretiens sur la pluralité des mondes, 1686), pour donner à leur création toutes les apparences de mondes réels, et nourrir à la fois la réflexion sur l'État idéal et la critique implicite de la France de Louis XIV. L'enjeu politique d'une telle production ne fait en effet pas de doute, et l'auteur s'inspire ici à juste titre des travaux menés par et autour de Monika Neugebauer-Wölk sur la politisation de l'utopie. Le récit de voyage utopique propose un véritable contre-modèle de société et d'organisation politique auquel Dietz consacre son premier chapitre, qui à lui seul représente plus du tiers de l'ouvrage. Ce déséquilibre dans l'économie générale de l'ouvrage aurait d'ailleurs dû être corrigé lorsque l'auteur a repris le manuscrit de sa thèse pour en tirer un volume de 204 pages de textes, notes comprises. Rappelant avec Myriam Yardeni que « la hiérarchie, l'architecture, l'harmonie, la normalité d'État louis-quatorzien deviennent objet de révolte », Dietz entreprend de répertorier l'ensemble des thèmes présents dans ces récits de voyages utopiques. Chacun d'entre eux est l'objet d'un développement nourri de citations abondantes, extraites des principaux titres mentionnés plus haut, qui sont d'un grand intérêt pour le lecteur français. Malheureusement, l'articulation entre les entrées thématiques est insuffisante ; aussi le lecteur doit-il, pour se faire une idée des principaux récits mobilisés par Dietz, en recomposer la trame en rapprochant les citations extraites de chacune d'entre elles. Si le plan thématique peut se justifier, en revanche une présentation préalable, même rapide, des principales sources et des utopies qu'elles construisent, s'imposait. Cette critique émise, l'ouvrage est riche en informations. Le récit de voyage utopique part à la recherche d'un homme nouveau, même s'il admet qu'il n'est plus possible à l'Européen de « redevenir huron ». La tentative de localiser les préadamites est d'autant plus pressante. La Terre australe de Gabriel de Foigny est peuplée d'hermaphrodites, manière de rétablir l'égalité parmi les humains. On retiendra également l'importance du thème religieux, qui met l'accent sur la nécessaire tolérance, le respect de l'autre, la liberté de conscience, et la religion naturelle. Fontenelle estime que « les Ajaoiens se croient donc fondés en raison pour mettre la Nature à la place de ce que nous nommons Dieu ». Dans cette recherche d'ordre harmonieux et d'une communication fluide et apaisée en « société », l'avocat Claude Gilbert, auteur de l'Histoire de Calejava ou de l'île des hommes raisonnables, envisage la possibilité pour l'épouse du principal protagoniste du dialogue de passer chaque semaine du catholicisme au protestantisme. De la confrontation entre la religion chrétienne et les croyances indiennes émergent chez le baron de Lahontan un authentique relativisme et l'idée d'un Dieu créateur et ordonnateur de la nature : «Nous reconnaissons un créateur de l'Univers, sous le nom de grand esprit ; ou de maître de la vie. » Avec Tyssot de Patot, Lahontan conteste la Bible : Dieu n'a pu vouloir la division des chrétiens, la Bible est donc une œuvre humaine, et par-là même faillible. La prégnance des débats contemporains autour du Tractatus theologico-politicus de Spinoza (1670) et de l'Histoire critique du Vieux-Testament de Richard Simon (1685) est manifeste. 
La recherche d'un État idéal a également sa traduction économique avec la critique du régime de propriété privée. Les Sévarambes de Veiras ont aboli la propriété privée et du même coup les risques de convoitises. Les hermaphrodites de La Terre australe limitent leur consommation au strict nécessaire, réglant ainsi la question des excédents et de leur appropriation. Les journées sont divisées en trois temps égaux : travail, plaisir, repos. Sans surprise, l'habitat est harmonieux parce que identique pour tous et géométrique - on aurait ici apprécié que Dietz dépasse le niveau de la description pour rapprocher ces écrits des débats autour de l'architecture utopique. Dans le contexte du colbertisme, les auteurs de ces écrits insistent sur l'importance d'une connaissance précise des effectifs des populations et des productions pour assurer une répartition équitable des ressources. La consanguinité est interdite, l'âge du mariage est contrôlé, afin d'assurer la robustesse des individus. «On ne voit chez eux ni langoureux, ni mutilés, ni refrigidis et maleficiatis », écrit Fontenelle dans l'Histoire des Ajaoiens. Avec le Fénelon des Aventures de Télémaque, les auteurs étudiés par Dietz soutiennent que la puissance se mesure davantage à la prospérité des peuples qu'aux territoires envahis. La critique de la monarchie louis-quatorzienne est ici manifeste. Chez les Sévarambes, l'autorité appartient au Soleil. Sevaris, qui préside de manière vertueuse aux destinées du peuple, ne saurait donc être que « vice-roi du Soleil ». Il refuse d'ailleurs toute appropriation de l'autorité divine et toute identification au dieu Soleil. Le prince doit maîtriser ses passions pour que ses sujets ne cèdent pas aux vices.

De fait, une des dimensions essentielles de ces récits qui s'inscrivent selon Dietz dans la recherche à la fois d'un art et d'une science du gouvernement, c'est bien évidemment, la tentative d'élaborer un ordre politique solide et harmonieux, favorisant l'épanouissement individuel et l'intérêt général. Les Ajaoiens sont égaux, car tous susceptibles de participer au gouvernement collégial. La peine de mort est dénoncée comme inhumaine par Tyssot de Patot qui soutient que « chaque homme, lorsqu'il s'agit de former une société, peut transférer à un autre, comme à un prince ou souverain, le droit et l'autorité que la nature lui a donnés sur lui-même, mais [qu'] il ne peut pas lui donner aucune puissance sur sa vie ». Dans un système où chacun est éduqué à la vertu et au désintéressement, la honte est une punition très efficace. Le travail peut également représenter une juste réparation du préjudice subi.

Dietz complète son étude en reliant de manière convaincante le choix d'écriture d'un récit de voyage en utopie, son impression et sa circulation aux questions du fonctionnement de la librairie, c'est-à-dire de la censure, à la diffusion des manuscrits philosophiques clandestins et des fictions libertines. Comment communique-t-on dans la France des années 1700 ? Reprenant à son compte les «libertine strategies » de Joan De Jean, et l'étude de Perez Zagorin sur Ways of lying. Dissimulation, persecution and conformity in Early Modern Europe, Dietz montre que les récits de voyages utopiques sont l'objet d'appropriation et de lectures multiples, investis par des stratégies de communication subversives, notamment dans la sphère du libertinage érudit. Au total, si la mise en perspective historique de la thèse et sa réécriture auraient sans doute mérité d'être approfondies, Dietz nous livre un ouvrage solide qui exhume des textes souvent méconnus.

Pierre-Yves BEAUREPAIRE 
André CHARRAK, Raison et perception. Fonder l'harmonie au XVIII siècle. Paris, Vrin, 2001. 13,5 × 21,5, 320 p., bibliogr., index (Mathesis).

Comme le montre Catherine Kintzler dans Jean-Philippe Rameau. Splendeur et naufrage de l'esthétique du plaisir à l'âge classique (Paris, Minerve, 1988), la musique devient au XVIII ${ }^{\mathrm{e}}$ siècle une «affaire d'État» qui mobilise philosophes et mathématiciens et s'amplifie à l'occasion des deux controverses majeures que sont la querelle des Lullistes puis, à partir de 1752, celle des Bouffons. Au centre de ces débats, l'œuvre musicale de Rameau, et plus encore sa tentative de fonder en nature le système de l'harmonie et le sentiment qui nous la manifeste. C'est à cette construction que s'attache d'abord l'ouvrage dense d'André Charrak, tiré d'une thèse de doctorat en philosophie. En rendant compte de l'œuvre de systématisation accomplie par Rameau et par là des nouveaux rapports qui s'établissent entre musique et sciences, l'auteur restitue aux nombreux débats de l'époque leur dimension tant épistémologique qu'esthétique et anthropologique. Ainsi, les Éléments de musique de d'Alembert sont plusieurs fois réédités, mais aussi augmentés en 1762 d'une préface par laquelle l'auteur s'en prend désormais ouvertement aux ambitions affichées par Rameau. Rousseau voit de son côté dans la théorie de Rameau, qui cherche à constituer la musique en une science physico-mathématique, l'une des manifestations du rationalisme ambiant qui s'efforce de «matérialiser » toutes les opérations de l'âme. Ce système serait volontairement « inintelligible », exclusivement réservé aux savants, et incapable de rendre raison de l'expérience sensible et du plaisir musical (Jean-Jacques Rousseau, Examen de deux principes avancés par M. Rameau, in Euvres complètes, vol. V, Paris, Gallimard (Bibliothèque de la Pléiade), 1995, p. 364 et Fragment, in ibid., p. 368). La controverse entre les deux auteurs, habituellement réduite à la question de la primauté de la mélodie ou de l'harmonie, se trouve ainsi renvoyée par Charrak à ses fondements et aux difficultés d'ordre philosophique engendrées alors par les questions musicales.

En effet, comment rendre compte du décalage éventuel entre l'étude des rapports qu'entretiennent les sons et le plaisir musical ; quelle place les principes de la théorie harmonique occupent-ils dans la classification encyclopédique des sciences ; comment caractériser enfin la reprise, par les auteurs du siècle des Lumières, de l'interrogation classique concernant les relations entre les mouvements du corps et les sentiments ? Ainsi, pour éclairer les choix philosophiques engagés par une théorie musicale, l'auteur se livre à l'exposé du système ramiste, qui occupe l'essentiel de la première partie de l'ouvrage. Les discussions soulevées par les tentatives de Rameau pour fonder la théorie harmonique sur le phénomène de la résonance du corps sonore imposent « d'interroger les conditions d'une application légitime des mathématiques, puis de la physique, aux ressources musicales. Il s'agit [...] d'envisager les rapports qu'entretiennent une formalisation largement inédite de la théorie harmonique, une physique de la vibration dont la mathématisation connaît d'importants progrès au milieu du siècle, et une théorie de la perception qui illustre le rapport des penseurs des Lumières avec la tradition classique » (p. 8). Le plan de l'ouvrage, nettement structuré, suit alors au plus près les difficultés engendrées par la tentative de fonder en raison la théorie harmonique : «Les objets de la théorie 
harmonique soulèvent ainsi le problème des relations entre la raison, qui s'engage dans l'appréhension des rapports des sons, et la perception, où s'éprouve le sentiment de l'harmonie » (p. 15).

Il faut rappeler la célèbre définition proposée par Rameau dans la Génération harmonique : «La musique est une science physico-mathématique, le son en est l'objet physique, et les rapports trouvés entre les différents sons en sont l'objet mathématique » (p. 149). Rameau conçoit à juste titre sa théorie harmonique comme une œuvre d'unification et de systématisation : elle devient « la science du monde la plus simple et la plus naturelle » (p. 39), par la découverte du principe à partir duquel peuvent se déduire tous les phénomènes musicaux.

Prolongeant le principe d'analogie entre les longueurs de corde, susceptibles d'une expression arithmétique, et les relations des sons entre eux, étudié par René Descartes dès 1618 et approfondi ensuite par Joseph Sauveur, Jean-Philippe Rameau accomplit le passage d'une théorie de la consonance à une théorie de l'accord : en tant que structure ou totalité (p. 34), l'accord est premier par rapport aux sons et aux intervalles qui le composent. Cette définition théorique de l'accord comme unité synthétique, irréductible à l'agrégation des parties, pose toutefois le problème, qui sera longuement développé dans la seconde partie de l'ouvrage, de son appréhension au niveau de la perception.

L'acoustique, à partir de 1726, vient apporter une confirmation expérimentale à l'entreprise d'unification opérée par Rameau depuis le Traité de l'harmonie, sans pour autant remettre en cause le recours aux spéculations arithmétiques plus traditionnelles. La définition du son musical tirée de la résonance du corps sonore prend ainsi place à l'intérieur de la théorie harmonique. La Génération harmonique fait alors l'objet d'une analyse exhaustive. Ce dernier texte élargit le domaine d'application des spéculations physiques, mais révèle aussi l'ambiguïté de la référence ramiste à l'acoustique expérimentale : «Rameau impose aux données acoustiques le filtre de son ancienne classification des consonances » (p. 116).

La première partie de l'ouvrage éclaire donc la compatibilité partielle des aspects mathématique et physique en fonction desquels Rameau entreprend d'exposer la musique comme une science. Le concept de génération harmonique engage en effet des registres théoriques variables, comme le montrent les riches analyses consacrées à l'explication de l'accord majeur et plus encore, à celles du mode mineur et de la dissonance. Ainsi cette dernière, qui ne peut être donnée dans l'expérience résonantielle, ne peut-elle relever que d'une génération indirecte, elle convoque «l'instinct» de l'auditeur, constitué par l'habitude de la résonance, thèse en laquelle se manifeste l'influence de Jean-Jacques Dortous de Mairan sur Jean-Philippe Rameau. L'écart entre les données empiriques et les configurations musicales contraint Rameau à en appeler aux capacités de l'auditeur pour expliquer ce que ne donnent pas effectivement les expériences de résonance. Poser un rapport de signe acoustique à interprétation harmonique préserve l'objectivité de l'ordre musical, garantie par le terme de nature, en laquelle il faut comprendre aussi bien les phénomènes sonores que les déterminations de la perception musicale : « [...] du phénomène acoustique à l'écoute spontanée, une même nature est à l'œuvre» (p. 132). Il ne s'agit donc pas simplement d'identifier des «faiblesses 
conceptuelles manifestes » (p. 289), mais bien de caractériser la théorie ramiste de l'harmonie par la diversité des opérations qu'elle légalise et d'y repérer la source d'une interrogation féconde.

À partir de ces précisions s'ordonnent les enjeux, distincts, des multiples oppositions qu'elle a suscitées. Nous n'en retiendrons que deux. L'absence d'une réduction effective des phénomènes de résonance aux principes mathématiques conduit notamment d'Alembert à n'envisager que la fondation expérimentale de la théorie musicale et à lui dénier toute prétention démonstrative. Les rapports mathématiques ne sont pas constitutifs de l'objet esthétique. L'acoustique musicale entre dans la catégorie des sciences conjecturales où seul l'emploi de raisonnements d'analogie et de convenance est permis. L'article «Fondamental », rédigé pour l'Encyclopédie ainsi que la seconde préface des Éléments de musique rendent parfaitement compte de la dimension épistémologique de la dispute et du revirement de d'Alembert à l'égard du théoricien autrefois salué comme le «Descartes de la musique ». Face à Rameau, Rousseau défend ensuite une généalogie de l'expression musicale qui engage l'ensemble de son système philosophique. Aussi est-ce moins l'harmonie que le processus d'harmonisation, terme d'une histoire, qu'il combat, ainsi que la confusion opérée entre l'analyse acoustique et l'argumentation esthétique, entre plaisir de sensation et affections morales que seule la mélodie, par sa fonction imitative, peut provoquer. En faisant de l'harmonie l'unique fondement de l'art, Rameau confondrait droit et fait, artifice et nature, mais aussi calculs abstraits et expérience sensible.

La seconde partie de l'ouvrage s'organise en trois moments qui rendent compte des modèles d'élucidation du sentiment de l'harmonie, sentiment en lequel on doit voir une épreuve pour les doctrines empiristes : la raison est-elle déjà à l'œuvre, sous une forme éventuellement cachée, dans l'expérience perceptive dont elle est censée dériver? L'appréciation esthétique requiert-elle qu'un jugement s'applique aux données sensorielles ? Selon Rameau, la perception elle-même est une représentation. Elle implique une mise en forme des données acoustiques, qui reste toutefois naturelle car les modifications que l'écoute apporte aux données acoustiques, loin d'introduire un point de vue relativisant, reposent elles-mêmes sur des dispositions physiologiques qui relèvent d'un même ordre mécanique. Face à ce traitement du problème de la perception, qui impute au sentiment de l'harmonie le statut d'une impression passive, garante de l'objectivité des rapports harmoniques prescrits par la nature, une autre position se développe, défendue par Diderot. Le principe de la perception des rapports, exposé pour la première fois dans les Principes généraux d'acoustique, s'inscrit dans une généalogie empiriste des connaissances humaines. Il présente, notamment dans ses versions ultérieures, l'intérêt de formuler un jugement sur l'organisation interne de la musique moderne qui dépasse les apories liées à l'idée d'imitation. Toutefois, en plaçant la cause du plaisir, non plus dans l'effet physique produit par la superposition des vibrations, mais dans l'appréhension, par l'esprit qui éprouve ce sentiment, des rapports qu'entretiennent les sons, il suscite de nouvelles objections quant au fonctionnement de la perception : il néglige l'hétérogénéité du raisonnement mathématique et du processus perceptif (d'Alembert), il requiert l'intervention d'un jugement dans 
l'élaboration d'une affection sensible (Rousseau), et pour finir renvoie aux difficultés gnoséologiques soulevées par la théorie classique des jugements naturels (p. 189).

Les tentatives pour fixer le sentiment du beau au plan de l'immanence sensorielle qu'André Charrak explore selon une double perspective : la théorie du sentiment intérieur développée par Francis Hutcheson, et les hypothèses mécaniques relatives à la structure et au fonctionnement de l'oreille, qui s'efforcent de rabattre la cause du plaisir sur les raisons de l'impression, et auxquelles Jean-Philippe Rameau emprunte l'idée d'une identité de structure entre le corps sonore et l'oreille -, par les méthodes analytiques qu'elles mettent en œuvre, enregistrent un décalage entre les propriétés structurelles de l'objet musical et son mode d'appréhension.

L'ouvrage souligne ainsi pour finir l'originalité de la synthèse opérée par Rousseau, qui permet de dépasser l'antinomie de la passivité et de l'activité et s'incarne dans le principe de «l'unité de mélodie ». La perception de l'harmonie est habituellement négligée par les commentateurs qui se concentrent sur la genèse et la communication des affections morales. L'originalité de la démarche ici suivie consiste à s'intéresser, non pas à l'esthétique authentique développée par Rousseau, mais précisément aux ressources matérielles, «physiques », qui doivent servir d' " occasion » aux passions de l'âme et semblent pouvoir susciter, au niveau perceptif, une forme de synthèse. C'est dans le temps que l'unification vécue des éléments de l'accord s'effectue : au sein d'une durée musicale, l'auditeur peut réaliser la synthèse des relations synchroniques qui définissent cet objet. La perspective esthétique inverse ainsi les termes de l'analyse physiologique : ce n'est plus l'organe qui est fait pour la musique, c'est la musique qui forme l'oreille. Cette étude de la théorie de la réceptivité selon laquelle le niveau de la sensation, où s'éprouvent les relations harmoniques, tolère déjà une mise en forme de l'impression, constitue l'un des moments forts de l'ouvrage. Comme ce dernier le montre très bien, les grands auteurs du XVIII ${ }^{\mathrm{e}}$ siècle se situent en retrait des grands systèmes métaphysiques du siècle précédent : pour Rousseau, l'action de l'âme sur le corps constitue bien « l'abîme de la philosophie » (voir également le passage significatif relevé dans les Euvres complètes, op. cit., vol. IV, p. LXXX), mais il n'en existe pas moins chez lui un intérêt évident pour le retentissement sur l'âme des phénomènes physiques, particulièrement fructueux dans les textes relatifs à la musique.

Ce livre, appuyé sur une très large connaissance des auteurs classiques, va donc bien au-delà d'une exposition du système ramiste lorsqu'il explique le réaménagement de la théorie musicale au siècle des Lumières. Il donne également à voir combien les contributions de Descartes, en particulier, structurent les débats relatifs à la musique et au problème de la perception au XVIII ${ }^{\mathrm{e}}$ siècle. Il offre ainsi une approche d'ensemble de la théorie de la connaissance en laquelle on peut suivre plusieurs glissements significatifs: les questions métaphysiques se transportent dans une histoire des connaissances humaines, le problème de l'union de l'âme et du corps, réputé insoluble, voit ses déterminations précises reportées dans l'examen des relations entre raison et perception. 
Michel BRIX, L'Héritage de Fourier. Utopie amoureuse et libération sexuelle. Jaignes, La Chasse au snark, 2001. $13 \times 17,224$ p., bibliogr. (Essais critiques).

Dans leur passage en revue des systèmes socialistes, Bouvard et Pécuchet résumaient le fouriérisme ainsi : «Toute femme, si elle y tient, possède trois hommes, le mari, l'amant et le géniteur. Pour les célibataires, le Bayadérisme est institué. — Ça me va ! dit Bouvard ; et il se perdit dans les rêves du monde harmonien. [...] Des navires traverseront les mers polaires dégelées sous les aurores boréales - car tout se produit par la conjonction des deux fluides mâle et femelle, jaillissant des pôles - et les aurores boréales sont un symptôme du rut de la planète, une émission prolifique. - Cela me passe, dit Pécuchet. » Ce sont ces deux points de la théorie fouriériste qui ont toujours paru irrecevables : les rêveries cosmogoniques et les descriptions du nouveau monde amoureux. Mais alors que Bouvard se laissait séduire par les promesses de l'utopie amoureuse, Michel Brix se montre très sévère envers la pensée de Charles Fourier dans ce domaine. De son livre sur la théorie fouriériste de l'amour et sur son influence supposée sur les mœurs contemporaines, plusieurs lectures sont possibles, que l'on considère L'Héritage de Fourier. Utopie amoureuse et libération sexuelle comme une étude littéraire, un essai d'histoire des idées, ou un pamphlet anti-utopique.

Dans la première partie («Le fouriérisme en son temps »), Brix revient très rapidement sur la question de l'amour dans l'utopie et retrace les grandes lignes du projet de Fourier, avant de se concentrer sur les théories concernant les passions et l'organisation des rapports sexuels en Harmonie, et de formuler sa thèse centrale selon laquelle la pensée de Fourier est le lieu d'une tension entre deux formes d'amour : le mysticisme et le libertinage. Cette opposition serait caractéristique de l'utopie en général et trouverait son origine dans un dualisme mis en place par la philosophie platonicienne et propagé par les écrivains romantiques. Dans la perspective d'une critique thématique de ce «dualisme amoureux », et s'appuyant sur des analyses développées dans son Éros et littérature. Le discours amoureux en France au XIXe siècle (Louvain, Peeters, 2001), Brix veut mettre en évidence dans cette dialectique de l'amour pur et de l'amour charnel les « liens profonds qui unissent les théories de Fourier - y compris les plus extravagantes - avec l'idéologie romantique » (p. 65). La somme érudite annonçait l'essai critique puisque dans le bref passage d'Éros et littérature où Fourier était mentionné, Brix citait des « déclarations où se devinent déjà les chemises bariolées et les barbes fleuries de nos gourous soixante-huitards » (p. 152). (Passons sur le cliché, et notons l'injuste double peine infligée aux auteurs des déclarations incriminées : dans Éros et littérature, Claire Démar et Pauline Roland, «saint-simoniennes convaincues, paient de leur personne pour la promotion de cette morale nouvelle »-1' « enseignement moral dissolu» de Prosper Enfantin; dans L'Héritage de Fourier (p. 75), ces mêmes martyres de l'utopie «payèrent de leur personne pour la promotion des idées fouriéristes ».)

Dans la seconde partie («Le meilleur des mondes amoureux ?»), l'auteur établit plus ou moins directement une sorte de filiation des héritiers de Fourier. Sont évoqués successivement l'engouement d'André Breton pour Fourier, l'œuvre 
de David Herbert Lawrence, les théories de Wilhelm Reich, le situationnisme et la pensée 68. Selon l'hypothèse de Brix, la loi du désir des surréalistes, la conscience sanguine de Lawrence, l'énergie vitale cosmique de Reich, les excès de l'hédonisme des années 1960, et la dictature contemporaine de la pansexualité ne seraient que des avatars de la théorie fouriériste de l'amour. L'évolution du dualisme amoureux conduit à une résolution de l'opposition en faveur de l'un de ses termes (le «mysticisme charnel »); mais, en fait de libération sexuelle, d'épanouissement de l'individu, d'émancipation de la femme, etc., il ne s'agirait que d'un simple renversement de l'oppression de «l'idéal angélique » en domination de « l'idéal libertin» (p. 201-202). Voilà donc, selon Brix, le résultat de la réalisation des promesses de Fourier.

À la question de savoir si l'établissement d'un «phalanstère global » a contribué à épurer les mœurs, le discours du citoyen de Namur répond par la négative. Soutenue par un ton parfois véhément, et malgré quelques formules artificiellement intempestives, et la reprise d'une antienne trop convenue à propos des « errances de l'utopie », la démonstration vaut la peine d'être suivie ; reste cependant à savoir si ses prémisses sont exactes, et si sa conclusion est probante. Les idées de Fourier ont-elles été effectivement réalisées ? Le rapport de l'utopie au platonisme et la stratégie utopique d'exclusion du Mal sont-ils vraiment ce que Brix en dit ici ? Tout cela serait sans doute à discuter plus longuement et plus précisément.

Quelle place pour les passions dans l'utopie ? Dès la préface de 1841 à la Théorie des quatre mouvements, les disciples de l'École sociétaire contestaient les arguments de «l'immoralité prétendue de la théorie de Fourier » : «Fourier veut que l'homme lâche la bride à ses passions », « Fourier attaque avec une audace inouïe et la morale et les moralistes », « Fourier propose des coutumes amoureuses qui sanctionneraient des relations réprouvées par la morale » (Charles Fourier, Théorie des quatre mouvements et des destinées générales, suivi de Le Nouveau Monde amoureux, Dijon, Presses du réel, 1998, p. 556). Et, Fourier lui-même, qui «manie la dialectique avec la même maîtrise que Hegel » (Friedrich Engels, Socialisme utopique et socialisme scientifique, 1880), avait perçu la dualité d'Éros (cynique/romanesque, sensuel/céladonien, etc.) et bien mesuré la difficulté de la question du rapport entre les sexes en utopie. Le Nouveau Monde amoureux comportait ainsi sa propre parade : « Des êtres tout cyniques, sensuels en amour, comme sont des civilisés, hommes et femmes, pourraient croire qu'en indiquant de nouveaux modes pour l'amour je ne vais travailler qu'à satisfaire leurs penchants grossiers ; [passage mangé par les souris] rien ; j'ai dit et je répète que le but du Créateur est d'établir la balance en un élément d'amour entre le matériel et le spirituel» (op. cit., p. 430). Fourier prévoyait que la réforme du régime des amours ne pourrait se faire que graduellement, lorsque les mauvaises habitudes seraient perdues, et peut-être seulement lorsque l'Harmonie serait déjà établie. Et en ce sens, l'essai de Brix serait une confirmation du pressentiment de Fourier qu'en Civilisation la liberté en amour ne pourrait être que galvaudée. Or, la fin de la théorie de l'attraction est l'association générale, en passions comme en industrie. Pour Fourier, c'est seulement dans l'harmonie entre le politique et le domestique que prend son sens le «rêve philosophique d'une grande famille de frères » (ibid., p. 496). 
Placé par Brix entre Pétrarque et Sade, Fourier se situe aussi entre Molière et Balzac, entre satire morale et histoire sociale (comme le dit Fredric Jameson dans «Ontology and Utopia », L'Esprit créateur, vol. XXXIV, 4, 1994). Replacé dans son contexte historique, Le Nouveau Monde amoureux apparaît comme une machine de guerre contre les contradictions d'une société (la Civilisation) régie en apparence par le principe de monogamie (voir Code Civil de 1804, livre I, titre V), et mue en secret par la polygamie furtive (voir Hiérarchie du cocuage). C'est cela le chaos des amours civilisées : l'amour égoïste, l'hypocrisie et l'impéritie générale sur les questions du sentiment. Et, selon Friedrich Engels, Charles Fourier fut « le premier à énoncer que, dans une société donnée, le degré d'émancipation de la femme est la mesure naturelle de l'émancipation générale ». Plutôt qu'une propension à un quelconque embrigadement érotique, on trouverait donc chez Fourier la lucidité du satiriste et le calcul raisonné d'une optimisation du jeu des passions - l'antithèse des drames bourgeois. «Un farouche républicain qui ne veut aimer qu'une seule [passage mangé par les souris] trouvera pleinement à se satisfaire dans l'harmonie et jouira d'une fidélité [mangé par les souris] garantie qu'elle ne l'est aujourd'hui, pourvu toutefois que la sienne soit [mangé par les souris], ce qui est assez rare chez tous ces champions de morale, qui sont d'ordinaire plus dépravés en secret que les francs libertins et se livrent cafardement aux adultères et fornications, stupres et autres goûts inconstitutionnels, tout en déclamant contre ceux qui avouent quelque papillonnage bien moins blâmable que le cynisme secret des moralistes » (Le Nouveau monde amoureux, op. cit., p. 431).

«L'actualité de Fourier» peut-elle se résumer à la réouverture d'un vieux procès et à ce nouvel acte d'accusation - l'effet néfaste qu' aurait eu au Xx siècle la théorie de l'attraction passionnée ? Dans «Vivre avec Fourier » (Critique, 281, 1970, repris in Sade, Fourier, Loyola, Paris, Seuil, 1971), Roland Barthes concluait $\mathrm{au}$ « caractère raisonnable [des] délires [de Fourier], puisque certains sont en voie d'application (accélération de l'Histoire, modification des climats par la culture et l'urbanisation, percée des isthmes, transformation des sols [...]) ». Et plus récemment, René Schérer versait au dossier des éléments qui attestent de cette actualité de l' « écosophie » de Fourier, en éditant ses textes Détérioration matérielle de la planète et De la médecine naturelle ou attrayante composée (Paris, EconomicaAnthropos, 2001). Reste donc à la fin ce constat, paradoxal certes, mais indépassable, et qu'il convient de rappeler toujours le plus équitablement possible, que le « caissier en délire » (Flaubert) est aussi un « rêveur sublime» (Stendhal). Comme le dit Pécuchet : "Qu'il y ait chez les utopistes, des choses ridicules, j'en conviens. Cependant, ils méritent notre amour. La hideur du monde les désolait, et pour le rendre plus beau, ils ont tout souffert. Rappelle-toi Morus décapité, Campanella mis sept fois à la torture, Buonarroti avec une chaîne autour du cou, Saint-Simon crevant de misère, bien d'autres. Ils auraient pu vivre tranquilles ! mais non! ils ont marché dans leur voie, la tête au ciel, comme des héros. » 
Michèle Riot-Sarcey, dir., L'Utopie en questions. Saint-Denis, Presses universitaires de Vincennes, 2001. 13,5 × 22, 261 p. (La Philosophie hors de soi).

«La mort annoncée des utopies semble susciter un regain d'intérêt pour un sujet depuis longtemps controversé » (p. 5). Dans son introduction à L'Utopie en questions, Michèle Riot-Sarcey résume bien les grandes lignes de ce recueil d'articles : en dépit de l'oubli dans lequel certains auraient aimé la voir tomber, de son exclusion de l'histoire et de la récurrence des critiques qui lui sont faites, l'utopie garde un attrait persistant, et soulève des questions, pertinentes aujourd'hui encore, sur l'impensé du politique, maintenant donc bien vivant le débat à son propos.

Les questions sur l'utopie posées par ce recueil d'articles prolongent des travaux développés dans le séminaire interdisciplinaire «De l'utopie et des utopistes » (université de Paris VIII), et s'inscrivent dans une série de publications récentes formée notamment par les livres de Riot-Sarcey, Le Réel de l'utopie. Essai sur le politique au XIXe siècle (Paris, Albin Michel, 1998), et de Miguel Abensour, L'Utopie de Thomas More à Walter Benjamin (Paris, Sens \& Tonka, 2000) et Le Procès des maîtres rêveurs suivi de Pierre Leroux et l'utopie (Arles, Sulliver, 2000). L'Utopie en questions se situe également dans la perspective générale esquissée par le numéro de la revue Raison présente consacré à l'utopie $(121,1997)$, d'où sont repris les articles de Jacques Rancière («Sens et usages de l'utopie »), de Miguel Abensour («Utopie et démocratie », qui conclut le recueil), ainsi qu'une version remaniée de l'article de Michael Löwy sur «L'utopie romantique de Walter Benjamin » (ici «L'utopie Benjamin »). Monique Boireau-Rouillé poursuit dans « Les enjeux politiques d'une science des sociétés » un travail présenté dans les actes du colloque qui s'est tenu à Besançon en 1997 (Utopies et sciences sociales, sous la direction de Bruno Péquignot, Paris, L'Harmattan, 1998). L'article de Michel Cordillot sur l'aventure américaine des fouriéristes offre une synthèse des passionnantes enquêtes «Autour de la colonie de Réunion, Texas », parues dans les Cahiers Charles Fourier (4, 1993).

C'est donc bien de la permanence du questionnement utopique que témoignent les treize articles que rassemble cet ouvrage. Si, comme le note Solange MercierJosa, « il y a reviviscence [des] discours "utopiques", sous différentes modalités pratiques, que ce soit à titre d'auxiliaires du mode de production capitaliste à l'intérieur même de celui-ci ou "d'espaces" de résistance à ce dernier et à la mondialisation du marché » (p. 131), L'Utopie en questions montre qu'il y a aussi une actualité de la réflexion théorique sur l'utopie, et rappelle l'importance de ses enjeux. Délimitée par des incursions dans le XVII ${ }^{\mathrm{e}}$ siècle (Eleni Varikas sur les utopies de la Révolution anglaise) et dans le $\mathrm{XX}^{\mathrm{e}}$ siècle (Michael Löwy sur Walter Benjamin, Jean-Marie Vincent sur Ernst Bloch et Theodor Adorno), L'Utopie en questions se présente d'abord comme un tableau de la recherche contemporaine sur les utopies du XIX ${ }^{\mathrm{e}}$ siècle, rendant compte de la diversité de ses approches, mais doit aussi se lire comme défense et illustration de l'utopie contre l'antiutopisme (c'est-à-dire contre le spécieux amalgame entre utopie et irréalisme ou totalitarisme). 
L'Utopie en questions comporte trois parties : « Figures de l'utopie, entre chimère et émancipation », «Traversées de l'utopie, entre science et critique sociales », «Enjeux et pratiques de l'utopie ». De l'une à l'autre, on retrouve des intérêts convergents, des problématiques communes, et des thèmes abordés sous divers angles : les critiques de l'utopie (Jean-Jacques Goblot, Miguel Abensour), le saintsimonisme (Monique Boireau-Rouillé, Antoine Picon, Jacques Rancière), l'utopie dans Le Curé de village d'Honoré de Balzac (Guy Rosa, Jacques Rancière), la question des rapports de l'utopie avec le romantisme (Guy Rosa, Michael Löwy), avec le marxisme (Solange Mercier-Josa, Michael Löwy), avec la science (Antoine Picon, Monique Boireau-Rouillé), et avec la démocratie (Miguel Abensour, Jacques Rancière), les problèmes de la place des femmes en utopie (Eleni Varikas, Michèle Riot-Sarcey) et de l'applicabilité de l'utopie (Michel Cordillot, JeanJacques Goblot).

Le point focal de ces réflexions est le rapport de l'utopie et de l'histoire. Comme l'annonce Michèle Riot-Sarcey dans l'introduction : « [...] la seule façon d'échapper aux troubles du contenu de l'utopie, c'est de quitter les chemins balisés des filiations fondées sur de simples analogies d'idées ou de genre. Afin d'atteindre l'historicité de l'utopie, à travers laquelle seulement il devient possible de redécouvrir le potentiel critique des projets, les conflits qu'ils engendrent, les travestissements qu'ils subissent, les réinterprétations qu'ils autorisent» (p. 7). «Effet d'utopie de la Révolution française », la philosophie de l'histoire a mis en avant la linéarité et la nécessité (François Hincker, p. 56-57). Le XIX siècle, « chrono-centriste » (Rosa, p. 31), marqué par «l'impensé téléologique qui anime le libéralisme » (BoireauRouillé, p. 85), et par l'idéologie du progrès, a historicisé les fictions utopiques. Or, critiquant cette « conception homogène, vide et mécanique du temps historique » (Löwy, p. 163), et un progrès menant à la catastrophe, Benjamin peut fournir un modèle pour une compréhension utopique du temps. Löwy montre en effet comment «Benjamin s'attaque à l'idéologie du progrès dans toutes ses composantes : l'évolutionnisme darwiniste, le déterminisme de type scientifico-naturel, l'optimisme aveugle» (p. 162). Cette critique vise également «l'évolutionnisme du "marxisme vulgaire" » (p. 163), ainsi que l' "optimisme téléologique de l'utopie blochienne qui lui donne une dimension acritique face aux catastrophes qui sont en train de se produire » (Vincent, p. 174). Pour Benjamin, le progrès est apocalyptique ; l'utopie pourrait être comprise comme l' « interruption d'une évolution historique menant à la catastrophe » (Löwy, p. 155).

« [S] l'inattendu, l'instantané peuvent être effacés de l'écriture de l'histoire, ils laissent subsister des traces "dans le souterrain" des idées émancipatrices » (RiotSarcey, p. 220). Aux croyances dans les déterminismes historiques doit être opposée une attention plus grande à la singularité des événements eux-mêmes - aux « fulgurances utopiques» (p. 215). Les articles d'Eleni Varikas («Coutume tyrannique, pourquoi obéir ? L'égalité des sexes dans l'utopie de la Révolution anglaise ») et de Michel Cordillot fournissent de bons exemples de ces manifestations utopiques : l'espace d'utopie qui s'est ouvert dans les années 1640-1650 en Angleterre, et les colonies fouriéristes et icariennes en Amérique dans la deuxième moitié du $\mathrm{XIX}^{\mathrm{e}}$ siècle. 
S'il faut bien constater la survivance des idées utopiques, que n'épuisent pas la série de leurs avatars, ni les relatifs échecs de leur mise en pratique, reste cependant que cette histoire souterraine peut difficilement éviter les « analogies d'idées ou de genre », et que l'étude de l'historicité de l'utopie reste en fin de compte indissociable d'une histoire de l'utopie. Si, par exemple, l'on évoque, comme Antoine Picon dans son article sur «La science saint-simonienne », le retour au cartésianisme opéré par Saint-Simon contre Newton, on peut entendre déjà dans La Nouvelle Atlantide de Bacon, et chez Condorcet, un appel pour une politique de la science fondée sur la coordination de la recherche scientifique. De la même façon, une réflexion sur la place des femmes dans l'utopie ne devrait pas passer trop vite sur L'Utopie de Thomas More. Lisant l'article « Femmes, défi des utopies », où RiotSarcey montre comment on a considéré que Thomas More avait «préservé la hiérarchie des sexes » (p. 212), on se rappelle un passage singulier - dans lequel on pourrait lire les prémices de la « vision plus égalitaire des rapports des sexes » qui se développera lors de la Révolution anglaise (Varikas, p. 192) : avant le mariage, « [1]a femme, qu'elle soit vierge ou veuve, par les soins d'une matrone vertueuse et sage, est exposée nue au prétendant et, par l'entremise d'un homme d'une probité éprouvée, le fiancé est à son tour montré nu à la jeune fille » (L'Utopie, traduction d'André Prévost, Paris, Mame, 1978, p. 550). Une coutume des Utopiens sur laquelle Francis Bacon avait d'ailleurs jeté un voile pudique. De la part du chancelier d'un roi qui se rendra célèbre par la répudiation de Catherine d'Aragon et par une politique matrimoniale des plus patriarcales (Henri VIII), écrire des Utopiens qu'ils n'admettent absolument pas qu' « une femme qui n'a commis aucune faute soit répudiée contre son gré » (L'Utopie, op. cit., p. 553) constituait assurément un petit fait non négligeable. Comme l'écrit Benjamin, dans Paris, capitale $d u X_{X} X^{e}$ siècle, "les rêves de l'avenir sont toujours "mariés" avec des éléments venus de l'histoire archaïque » (cité par Löwy, p. 158).

À l'issue de son récit des mésaventures des utopistes au Texas, Cordillot rappelle qu'en définitive ce sont les hommes et les femmes qui sont les acteurs de l'histoire. L'utopie s'incarne dans des histoires individuelles. Arsène Sauva, jeune tailleur parisien, s'installa en 1860 dans une colonie icarienne du Missouri, revint en France, adhéra à l'Association internationale des travailleurs, défendit la Commune, s'associa aux socialistes new-yorkais, rejoignit les icariens de l'Iowa, puis, de retour à Paris en 1880, se remit à son atelier. Riot-Sarcey dit à propos de Condorcet, qui conclut son Esquisse d'un tableau historique des progrès de l'esprit humain par un Fragment sur l'Atlantide, qu' « adossé à la mort, il [pressentit] cependant le devenir heureux de l'humanité »(p. 216). En 1940, peu avant de se suicider, Benjamin reprit une dernière fois ses thèses utopiques dans «Sur le concept d'histoire » (Löwy, p. 161). La mort annoncée suscite un regain d'intérêt pour l'utopie, et l'imminence de la catastrophe accroît l'urgence d'un tel questionnement. 